\title{
Errata
}

\section{Transition Metal-based Lewis Acid Catalyzed Ring Opening of Epoxides Using Amines Under Solvent-Free Conditions}

Pei-Qing Zhao, Li-Wen Xu, * Chun-Gu Xia* Synlett 2004, 846.

The schemes of entry 7 and entry 10 in Table 1 were incorrect, the correct figures are shown as follows:

Table 1 Ring Opening of Various Epoxides with Amines Catalyzed by $\mathrm{SnCl}_{4} \cdot 5 \mathrm{H}_{2} \mathrm{O}$ under Solvent-Free Conditions

Entry

